\title{
Gemeinsam an der IFAS 2018
}

\author{
Claudia Blackburna, Stephanie Alder \\ a Leiterin Dienstleistungen und Mitgliedschaft \\ b Verantwortliche Marketing und Kommunikation FMH Consulting Services AG
}
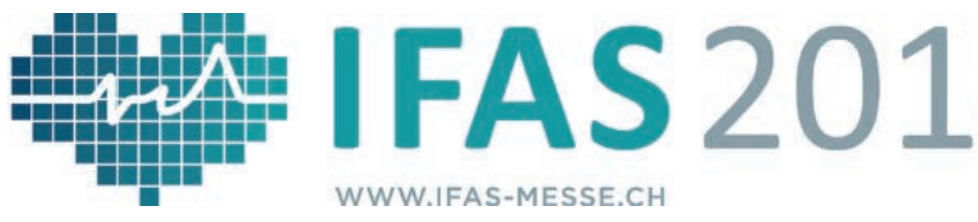

WWW.IFAS-MESSE.CH

\section{Workshops der FMH, der FMH Services und HIN am Donnerstag, 25. Oktober 2018}

Veranstaltungsort: Seminarraum K6 (Zugang vom Galeriegeschoss vor Halle 7).

Teilnehmerkreis: Die FMH- und FMH-Services-Workshops stehen ausschliesslich Ärztinnen und Ärzten sowie deren MPA offen (sofern die Anmeldung durch den Praxisinhaber erfolgt). Sie werden ausschliesslich in deutscher Sprache durchgeführt und sind kostenlos. Die HIN-Workshops richten sich an Ärztinnen und Ärzte, IT-Fachpersonen von Institutionen sowie HIN-Partner. Die Teilnahme an den Workshops berechtigt zum Gratiseintritt zur IFAS 2018.

Anmeldung: über das Online-Anmeldeformular unter www.fmhservices.ch

Workshop Nr. 1 (FMH): Aktuelle Fragen rund um die Krankengeschichte

Donnerstag, 25.10.2018, 10.00-10.45 Uhr

Referentin: Dr. iur. Caroline Hartmann, Rechtsanwältin,

Korrespondenz: FMH / Dienst DLM

Elfenstrasse 18

Postfach 300

CH-3000 Bern 15

Tel. 0313591111

dlm[at]fmh.ch
Stv. Leiterin Gutachterstelle FMH

Fokus auf:

- Einsichts- und Kopienrechte des Patienten an der Krankengeschichte

- Aufbewahrungspflicht

- Die Krankengeschichte als Beweismittel in Haftpflichtfällen
Workshop Nrn. 2 und 7 (HIN): IT-Security-Awareness-Schulung: Für grössere Institutionen und deren Gesundheitsfachpersonen Donnerstag, 25.10.2018, 11.00-11.45 Uhr und 16.00-16.45 Uhr Referenten: Lucas Schult, Leiter IT (CIO) und Stv. Geschäftsführer der Health Info Net AG (HIN)

Hacker-Attacken mit gravierenden Folgen wie Datenverlust, finanziellen Schäden oder Verletzung des Patientengeheimnisses treten immer häufiger auf. Daher ist es wichtig, gut ausgebildet zu sein und den eigenen Computer sowie die gesamte Institution umfassend zu schützen. Das Referat vermittelt wichtige Sicherheitsmassnahmen und Werkzeuge, um sich optimal vor den $\mathrm{Ge}-$ fahren aus dem Internet zu schützen.

Workshop Nrn. 3 und 6 (HIN): IT-Security-Awareness-Schulung: Für Ärzte, medizinische Praxisassistentinnen und kleinere Gesundheitseinrichtungen

Donnerstag, 25.10.2018, 12.00-12.45 Uhr und 15.00-15.45 Uhr Referent: Christian Greuter,

Geschäftsführer der Health Info Net AG (HIN)

Um Cyber-Attacken bestmöglich zu verhindern, hat IT-Security Awareness für Gesundheitsfachpersonen oberste Priorität. Dieses Referat vermittelt alle notwendigen Hintergründe und weist auf aktuelle Risiken im Bereich IT-Sicherheit und Internetkriminalität hin. DerWorkshop präsentiert Massnahmen zum Schutz von Systemen und zeigt auf, wie Ärztinnen und Ärzte sich und ihre Praxen optimal vor Angriffen schützen können.

Workshop Nr. 4 (FMH Services): Vermögensaufbau für Ärztinnen und Ärzte - welche Strategie soll ich verfolgen?

Donnerstag, 25.10.2018, 13.00 -13.45 Uhr

Referent: Alexander Bruderer, FMH Services (Insurance)

Häufig setzen sich Ärztinnen und Ärzte nur wenig mit der Strategie des Vermögensaufbaus auseinander. Sie führen üblicherweise ein 3.-Säule-Konto und sind bei der Pensionskasse angeschlossen. Was sind alternative Strategien, welche auch bei Invalidität für ausreichend Kapital sorgen? Welche Vermögensstrategie erlaubt die Aufrechterhaltung einer maximalen Flexibilität? Der Workshop soll zur Klärung dieser Fragen beitragen.

Workshop Nr. 5 (FMH Services): Rund um Verträge - Tipps für angestellte und selbständige Ärztinnen und Ärzte

Donnerstag, 25.10.2018, 14.00-14.45 Uhr

Referent: PatrickTuor, Leiter Beratung, FMH Consulting Services AG Verträge verschiedenster Art sind wichtige Bestandteile derTätigkeit als angestellter oder selbständig tätiger Arzt. Der Workshop vermittelt wichtige Informationen, die bei der Erstellung von Verträgen beachtet werden müssen.

- Arbeitsverträge

- Gesellschaftsverträge

- Übernahme- und Verkaufsverträge 